\title{
Correspondence
}

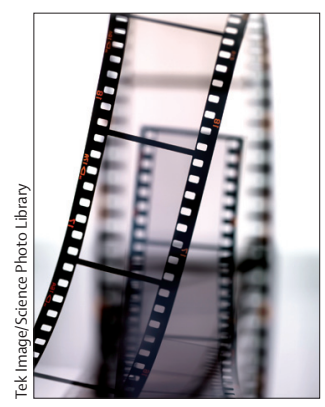

For more on The Global Health Film initiative see http://www. globalhealthfilm.org of disease conviction. We argue that the recognition of health anxiety and hypochondriasis is not just a "fashion in terminology" as Williams and House suggest, but an important and separate anxiety disorder that deserves its own classification, as indeed has happened recently with the introduction of illness anxiety as a diagnosis in DSM-5. ${ }^{4}$ The cognitive behavioural treatment of health anxiety is not just like any other, and it might be more appropriate for it to be initially given by nurses and other hospital staff in the clinics where the disorder presents rather than detected by screening or external referral, but this ultimately depends on better identification of the condition in hospital care, where much attention is needed. The disorder is not mild or trivial. It causes great suffering and tends to be persistent as well as complex, ${ }^{5}$ and the continued benefit of treatment after 2 years in our trial ${ }^{2}$ shows the value of a successful intervention, comparing very favourably with the long-term outcome of cognitive behavioural treatment for generalised anxiety disorder. ${ }^{6}$ We follow up the patients in this trial to establish whether benefits are still present 5 years after this brief form of treatment.

We declare that we have no competing interests.

*Peter Tyrer, Paul Salkouskis, Helen Tyrer, Simon Dupont, David Murphy p.tyrer@imperial.ac.uk

Centre for Mental Health, Imperial College, London W6 8LN, UK (PT, HT); Department of Psychology, University of Bath, Bath, UK (PS); Greenacres Centre, Hillingdon Hospital, Pield Heath Road, Uxbridge, UK (SD); and Department of Clinical Psychology, Charing Cross Hospital, Fulham Palace Road, London, UK (DM)

1 Williams C, House A. Cognitive behaviour therapy for health anxiety. Lancet 2014; 383: 190-91.

2 Tyrer P, Cooper S, Salkovskis P, et al. Clinical and cost-effectiveness of cognitive behaviour therapy for health anxiety in medical patients: a multicentre randomised controlled trial. Lancet 2014; 383: 219-25.

3 Clark DM, Salkovskis PM, Hackmann A, et al. Two psychological treatments for hypochondriasis: a randomized controlled trial. BrJ Psychiatry 1998; 173: 218-25.

4 American Psychiatric Association. Diagnostic and Statistical Manual of Mental Disorders, 5th edn. Washington, DC: APA, 2013: 315.
5 Seivewright $\mathrm{H}$, Salkovskis $\mathrm{P}, \mathrm{Green}$ J, et al. Prevalence and service implications of health anxiety in genitourinary medicine clinics. Int J STD AIDS 2004; 15: 519-22.

6 Durham RC, Higgins C, Chambers JA, et al. Long-term outcome of eight clinical trials of CBT for anxiety disorders: symptom profile of sustained recovery and treatment-resistant groups. J Affect Dis 2012; 136: 875-81.

\section{Founding of the Global Health Film initiative}

Film plays a unique role in today's society. It is an art form, a language, an educational tool, a method of information delivery, and a vehicle for social marketing. Film has the power to communicate stories, to stir emotions, to inspire, to encourage action, and highlight inequities.

The Global Health Film initiative is a partnership between the Bill \& Melinda Gates Foundation, the Royal Society of Medicine, and the London School of Hygiene and Tropical Medicine. The primary objective of the Global Health Film initiative is to use film as a catalyst for discussion and for change in policy and practice for health worldwide.

Traditionally, film has been a powerful mechanism to communicate public health messages. In the late 1920s, DM Connan, Bermondsey's Medical Officer of Health, highlighted the importance of visual imagery and adoption of the latest technology to engage the public. Bermondsey Borough Council pioneered public health cinema to communicate messages on health and disease in one of London's most deprived areas. ${ }^{1}$

Our activities will include the establishment of an annual Global Health Film Festival from 2015, based at the Royal Society of Medicine in London with satellite screenings. The Lancet will be offering a prize for best documentary in global health and for best young filmmaker.

A film workshop will also bring together filmmakers, journalists, scientists, doctors, public health advocates, and those interested in film and in global health to combine the skills and the language of filmmaking with the technical knowledge of public health sciences and critical appraisal. We hope that this innovative approach will bring together some leading minds to better communicate the advances and challenges in global health to the public, health professionals, and policy makers.

In concert with the open access movement in medical publishing, we aspire for documentary film to join the open-access movement. The Global Health Film initiative will work with sector-leading organisations, film production companies, and funders to develop an online platform to share high quality documentary film on global health.

We urge the public health community to support the initiative by contacting the secretariat with nominations for the film festival and suggesting collaborative centres for satellite screenings. Exposure to public health information in the media has the potential to educate, empower, and inspire individuals to live healthier lives.

JRF is director of the Global Health Film initiative. LB is coordinator of the Global Health Film initiative. We declare that we have no competing interests.

\section{* Joseph R Fitchett,}

Lalitha Bhagavatheeswaran,

Maysoon Dahab, Andy P Haines, W John Edmunds, on behalf of the Global Health Film initiative

\section{joseph@filminitiative.org}

Department of Infectious Diseases, King's College London, London SE1 9RT, UK (JRF); Royal Society of Medicine, London, UK (LB, MD); Department of Population Health and Department of Social and Environmental Health, London School of Hygiene \& Tropical Medicine, London, UK (APH); and Department of Infectious Disease Epidemiology, London School of Hygiene \& Tropical Medicine, London, UK (WJE)

1 Wellcome Collection. Here Comes Good Health! London: Wellcome Trust. http://www. wellcomecollection.org/whats-on/exhibitions/ here-comes-good-health.aspx (accessed March 17, 2014).

2 Wakefield MA, Loken B, Hornik RC. Use of mass media campaigns to change health behaviour. Lancet 2010; 376: 1261-71. 